\title{
Synthesis and Characterization of New Side-Chain Liquid Crystalline Polyoxazolines
}

\author{
Kyung-Min KIM, Yusuke ImaI, Kensuke NAKA, and Yoshiki CHUJO ${ }^{\dagger}$ \\ Department of Polymer Chemistry, Graduate School of Engineering, Kyoto University, \\ Yoshida, Sakyo-ku, Kyoto 606-8501, Japan
}

(Received December 14, 1999)

\begin{abstract}
Side-chain liquid crystalline (LC) polyoxazolines with different contents of mesogens and spacer lengths were synthesized by the reaction of partially hydrolyzed poly( $N$-acetylethylenimine)s with cyanobiphenyl mesogens having carboxylic acid group in the presence of DCC as a condensing agent. The obtained LC polyoxazolines seem to have broader LC phase than the corresponding cyanobiphenyl mesogenic compounds. The melting temperature and LC phase range decreased as the methylene units in the spacer increased. It was found that their liquid crystallinities were closely dependent on the degrees of substitution and the length of the spacer group incorporated into the portion of the ethylenimine. The critical percentage which the LC phase can be observed is above $27 \mathrm{~mol} \%$ of cyanobiphenyl moiety introduced to hydrolyzed polyoxazolines. From optical polarizing microscopy, the nematic texture of all the prepared cyanobiphenyl compounds changed into the smectic texture of the LC polyoxazolines except LC polyoxazolines with decamethylene spacer.

KEY WORDS Side-Chain Liquid Crystalline Polymers / Polyoxazoline / Cyanobiphenyl Mesogenic Group / Liquid Crystallinity/Smectic Texture /
\end{abstract}

Liquid crystalline polymers have been studied interestingly so far because of their unusual optical, electrical and mechanical properties. Many studies using these properties have centered on the application for a variety of practical devices such as reversible optical storage, liquid crystal display (LCD), and nonlinear optical media. ${ }^{1-3}$ Side-chain liquid crystalline polymers (SCLCPs), especially, were investigated intensively for several years because of the clearly established structureproperty relationship, ${ }^{4,5}$ that is to say, two more or less independent subsystems are formed; the mesogens, which are free to form LC-phases and the polymer chains, which may adapt a coil conformation. Following this concept a wide variety of SCLCPs was synthesized. SCLCPs exhibit the easiness to control $T_{\mathrm{g}}$ of the polymers with changing the length of flexible spacer which decouples the motions of side groups from that of the main chain incorporated into mesogenic group. ${ }^{6,7}$ Furthermore, they have a good solubility in common organic solvent, can be easily made to polymeric thin film, and show advantage to introduce various mesogenic groups into the side chain. ${ }^{8}$ They can be applied for optical data storage media and also used as the thin-layer film which LC material can be oriented. ${ }^{9,10}$

We have recently explored a wide variety of functional materials based on polyoxazolines. Such examples are polymeric surfactants, ${ }^{11-13}$ hydrogels, ${ }^{14-16}$ enzymemodifiers, ${ }^{17,18}$ and homogeneous hybrid materials ${ }^{19,20}$ at the molecular level combination. These functions are derived from interesting properties of polyoxazolines such as high hydrophilicity and good compatibility with several organic polar polymers. ${ }^{21,22}$ It should be also noted that the ring-opening polymerization of oxazoline proceeds in a living manner, which makes it possible to control the molecular weight of the obtained polymer, to obtain monodispersed polymer and also to design the polymer structure precisely.

\footnotetext{
${ }^{\dagger}$ To whom correspondence should be addressed.
}

Polyoxazoline is known to have a relatively low $T_{\mathrm{g}}$, and would be one of the good candidates as the main chain for SCLCPs. There was one study using these properties to make LC homopolyoxazolines. ${ }^{23}$ However, very littile is known about the preparation of partially hydrolyzed polyoxazoline (random copolymer) which is modified with different contents of mesogens and different spacer lengths. SCLCPs based on polyoxazoline are expected to show good affinity with organic polar polymers and also with inorganic materials such as silica or alumina. Here, we report in detail the results on the preparation and properties of the new side-chain liquid crystalline polyoxazolines containing different contents of mesogens which are cyanobiphenyl moiety and different spacer lengths. The number of methylene units in the spacer was varied from 2 to 6 in an even number, and 10 unit also was included. For convenience, LC polyoxazolines are denoted as Polyn- $m$, where $n, m$ describe the number of methylene units in the spacer and the percentage of the inserted cyanobiphenyl moiety, respectively in this paper.

\section{EXPERIMENTAL}

General Procedure

${ }^{1} \mathrm{H}$ NMR spectra were obtained on a $270 \mathrm{MHz}$ JEOLJNM-GX 270 NMR spectrometer. UV absorption spectra were obtained from a JASCO V-530 spectrophotometer. FT-IR spectra were recorded on a Hitachi 260-50 grating spectrophotometer. The thermal behavior was examined by DSC and TGA (Seiko Instruments Inc., Shimadzu DT-30) under a nitrogen atmosphere. The birefringent textures of the synthesized monomers and polymers were observed by a polarizing microscope (Nikon Type120) equipped with a hot stage.

\section{Materials}

All solvents and reagents were used as supplied except for the following materials. 2-Methyl-2-oxazoline 


\section{Scheme 1 .}

1)

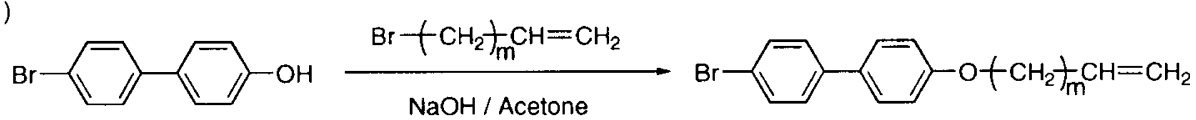

(1)

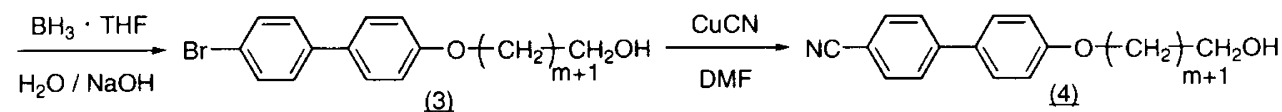

(3)

$(4)$

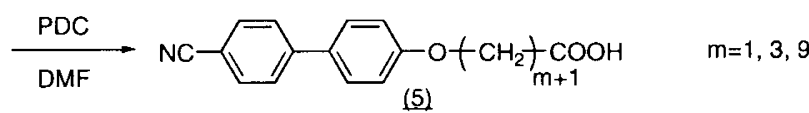

2)

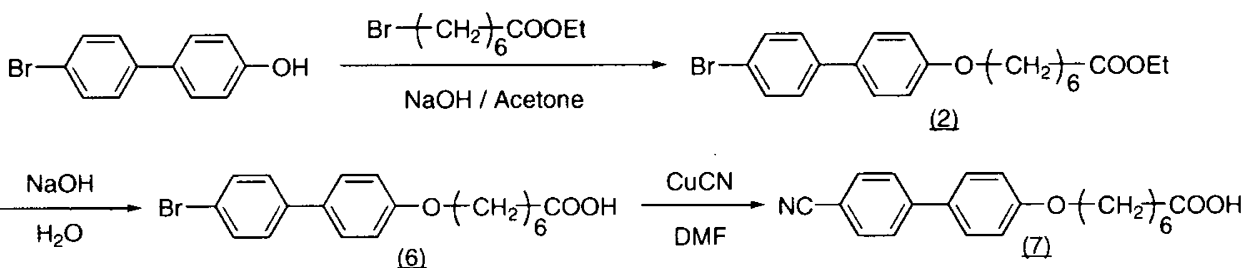

(Aldrich) was distilled from potassium hydroxide. Methyl $p$-toluenesulfonate was distilled under reduced pressure. $N, N$-Dimethylformamide (DMF) was dried over sodium hydride and freshly distilled under reduced pressure. Acetone was distilled from potassium carbonate under nitrogen. Acetonitrile and chloroform were distilled from calcium hydride and calcium carbonate, respectively. Tetrahydrofuran (THF) was distilled from sodium under nitrogen.

\section{Synthesis of Four Different Partially Hydrolyzed Polyoxazolines}

A series of the partially polyoxazolines with various degrees of hydrolysis were prepared by the ring-opening polymerization of 2-methyl-2-oxazoline followed by alkaline hydrolysis with controlled amount of $\mathrm{NaOH}$, according to the procedure reported before ${ }^{19,20}$ Before partially hydrolysis, the molecular weight of polyoxazoline was estimated by GPC analysis: $M_{n}=6300, M_{w}=10300$, $M_{n} / M_{w}=1.64$ (polystyrene standard; eluent, DMF).

\section{Synthesis of Cyanobiphenyl Mesogenic Compounds}

Synthesis of 5-(4-Bromobiphenyloxy)-1-pentene (1). A mixture of $\mathrm{NaOH}(1.69 \mathrm{~g}, 0.045 \mathrm{~mol})$ and 4-(4bromophenyl)phenol (10 g, $0.040 \mathrm{~mol})$ was dissolved in distilled acetone $(60 \mathrm{~mL})$. The mixture was stirred, and 5-bromo-1-pentene $(5.87 \mathrm{~mL}, 0.042 \mathrm{~mol})$ was added slowly. The reaction mixture was refluxed for $24 \mathrm{~h}$. After evaporation of the solvent, the crude mixture was washed with distilled water to remove the salts and dried in vacuo. The white crude product was recrystallized from methanol $(10.3 \mathrm{~g}, 81 \%), \mathrm{mp} 122-123{ }^{\circ} \mathrm{C} .{ }^{1} \mathrm{H}$ $\mathrm{NMR}\left(\mathrm{CDCl}_{3}\right): \delta 7.55-7.38(\mathrm{~m}, 6 \mathrm{H}), 6.98(\mathrm{~d}, 2 \mathrm{H}), 5.94-$ $5.79(\mathrm{~m}, 1 \mathrm{H}), 5.11-4.99(\mathrm{t}, 2 \mathrm{H}), 4.01(\mathrm{t}, 2 \mathrm{H}), 2.3(\mathrm{q}, 2 \mathrm{H})$, $1.96(\mathrm{q}, 2 \mathrm{H})$. Other vinyl compounds with different length of methylene spacer were also prepared by the same procedure.

Synthesis of Ethyl 7-(4-Bromobiphenyloxy)heptanoate
(2). Ethyl 7-(4-bromobiphenyloxy)heptanoate was synthesized by using ethyl 7-bromoheptanoate without using vinyl compound according to the above procedure. Yield was $75 \%, \mathrm{mp} 107-108^{\circ} \mathrm{C} .{ }^{1} \mathrm{H} \mathrm{NMR}\left(\mathrm{CDCl}_{3}\right): \delta 7.54$ $-7.39(\mathrm{~m}, 6 \mathrm{H}), 6.97(\mathrm{~d}, 2 \mathrm{H}), 4.17(\mathrm{q}, 2 \mathrm{H}), 3.99(\mathrm{t}, 2 \mathrm{H})$, $2.34(\mathrm{t}, 2 \mathrm{H}), 1.81(\mathrm{q}, 2 \mathrm{H}), 1.67(\mathrm{q}, 2 \mathrm{H}), 1.54-1.42(\mathrm{~m}$, $4 \mathrm{H}), 1.28(\mathrm{t}, 3 \mathrm{H})$.

Synthesis of 5-(4-Bromobiphenyloxy)pentanol (3). Tetrahydrofuran (THF) was added in the 5-(4bromobiphenyloxy)-1-pentene (1) (4.2 g, $0.013 \mathrm{~mol})$ under nitrogen atmosphere, and cooled at $0^{\circ} \mathrm{C}$. To this solution was added the $\mathrm{BH}_{3}$. THF complex $(13.25 \mathrm{~mL}, 0.013$ mol). After the mixture was stirred for $1 \mathrm{~h}$ at room temperature, the sodium hydroxide $(0.51 \mathrm{~g}, 0.013 \mathrm{~mol})$ in water $(30 \mathrm{~mL})$ and hydrogen peroxide $(1.41 \mathrm{~mL}, 0.013$ mol) were added slowly in the sequence to watch out happening of hydrogen gas and heat. The resulting mixture was stirred at room temperature for $12 \mathrm{~h}$. After evaporation of the solvent, the white mixture was extracted with diethyl ether and washed with aqueous sodium carbonate solution. After being dried over sodium sulfate, the solution was concentrated and dried in vacuo to produce white solid $(4.91 \mathrm{~g}, 55 \%), \mathrm{mp} 141-$ $143^{\circ} \mathrm{C} .{ }^{1} \mathrm{H} \mathrm{NMR}\left(\mathrm{CDCl}_{3}\right): \delta 7.55-7.38(\mathrm{~m}, 6 \mathrm{H}), 6.98(\mathrm{~d}$, $2 \mathrm{H}), 4.03(\mathrm{t}, 2 \mathrm{H}), 3.7$ (q, 2H), 1.87 (q, 2H), 1.69-1.54 (m, $4 \mathrm{H}), 1.27(\mathrm{t}, 1 \mathrm{H})$. Other bromo biphenyl alcohols with different length of methylene spacer were also prepared by the same procedure.

Synthesis of 5-(4-Cyanobiphenyloxy)pentanol (4). (4) was synthesized similarly according to a literature procedure. $^{24}$ Yield was $60 \%, \mathrm{mp} 105-106^{\circ} \mathrm{C} .{ }^{1} \mathrm{H}$ NMR $\left(\mathrm{CDCl}_{3}\right): \delta 7.71(\mathrm{q}, 4 \mathrm{H}), 7.51(\mathrm{~d}, 2 \mathrm{H}), 6.97(\mathrm{~d}, 2 \mathrm{H}), 4.05(\mathrm{t}$, $2 \mathrm{H}), 3.7(\mathrm{q}, 2 \mathrm{H}), 1.87(\mathrm{q}, 2 \mathrm{H}), 1.69-1.54(\mathrm{~m}, 4 \mathrm{H}), 1.27(\mathrm{t}$, $1 \mathrm{H})$. Other cyano biphenyl alcohols with different length of methylene spacer were also prepared by the same procedure.

Synthesis of 5-(4-Cyanobiphenyloxy)valeric Acid (5). (5) was prepared similarly according to a literature pro- 


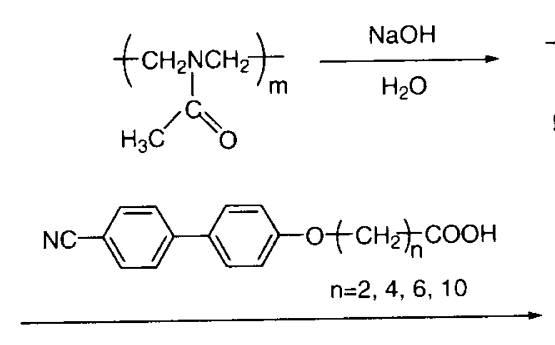

$\mathrm{DCC}, \mathrm{CHCl}_{3}$

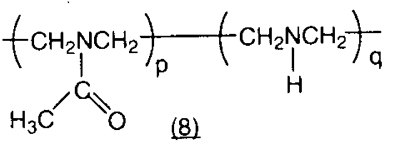

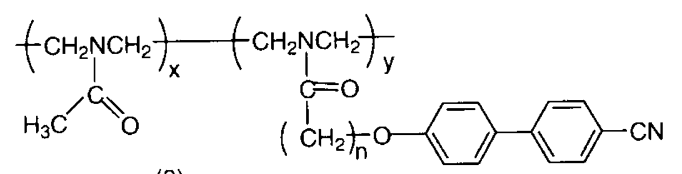

(9)

Table I. Preparation of LC polyoxazolines (9) with four methyl-

cedure. ${ }^{25}$ Yield was $41 \%$, mp $137-138^{\circ} \mathrm{C} .{ }^{1} \mathrm{H}$ NMR $\left(\mathrm{CDCl}_{3}\right): \delta 7.71(\mathrm{q}, 4 \mathrm{H}), 7.51(\mathrm{~d}, 2 \mathrm{H}), 6.97(\mathrm{~d}, 2 \mathrm{H}), 4.05(\mathrm{t}$, $2 \mathrm{H}), 2.50(\mathrm{t}, 2 \mathrm{H}), 1.89-1.86(\mathrm{~m}, 4 \mathrm{H})$. Other cyano carboxylic acids with different length of methylene spacer were also prepared by the same procedure.

Synthesis of 7-(4-Bromobiphenyloxy)heptanoic Acid (6). A mixture of ethyl 7-(4-bromobiphenyloxy)heptanoate (2) $(2.0 \mathrm{~g}, 0.005 \mathrm{~mol})$ and sodium hydroxide $(0.4 \mathrm{~g}$, $0.01 \mathrm{~mol})$ was dissolved in water $(100 \mathrm{~mL})$. The white suspension mixture was refluxed for $24 \mathrm{~h}$, cooled to room temperature, and diluted with water $(200 \mathrm{~mL})$. After removal of the insoluble parts by filtration, the mixture was acidified with concentrated hydrochloric acid $(10 \mathrm{~mL})$ under ice cooling. The white precipitate was collected by filtration, washed well with water, and dried in vacuo to produce a white solid $(1.6 \mathrm{~g}, 86 \%), \mathrm{mp} 143-$ $144{ }^{\circ} \mathrm{C} .{ }^{1} \mathrm{H} \mathrm{NMR}\left(\mathrm{CDCl}_{3}\right): \delta 7.55-7.38(\mathrm{~m}, 6 \mathrm{H}), 6.98(\mathrm{~d}, 2$ $\mathrm{H}), 4.03(\mathrm{t}, 2 \mathrm{H}), 2.4(\mathrm{t}, 2 \mathrm{H}), 1.87(\mathrm{q}, 2 \mathrm{H}), 1.69(\mathrm{q}, 2 \mathrm{H}), 1.55$ $-1.49(\mathrm{~m}, 4 \mathrm{H})$

Synthesis of 7-(4-Cyanobiphenyloxy)heptanoic Acid (7) . 7-(4-Cyanobiphenyloxy)heptanoic acid was prepared by using cupper cyanide with 7-(4-bromobiphenyloxy) heptanoic acid (6) according to the same procedure of making 5-(4-cyanobiphenyloxy)pentanol (4). Yield was $51 \%, \mathrm{mp} 118-119^{\circ} \mathrm{C}{ }^{1} \mathrm{H} \mathrm{NMR}\left(\mathrm{CDCl}_{3}\right): \delta 7.71(\mathrm{q}, 4 \mathrm{H})$, $7.51(\mathrm{~d}, 2 \mathrm{H}), 6.97(\mathrm{~d}, 2 \mathrm{H}), 4.03(\mathrm{t}, 2 \mathrm{H}), 2.41(\mathrm{t}, 2 \mathrm{H}), 1.84-$ $1.49(\mathrm{~m}, 6 \mathrm{H})$.

Synthesis of Polyoxazolines Having Cyanobiphenyl Mesogenic Compounds

Side-chain liquid crystalline polyoxazolines with different contents of mesogens and spacer lengths were prepared by the condensation reaction of partially hydrolyzed poly(2-methyl-2-oxazoline) and acid having cyanobiphenyl mesogenic moiety, as illustrated in Scheme 2. Details of synthetic procedure are described previously. ${ }^{19,20}$

\section{RESULTS AND DISCUSSION}

\section{Synthesis of Cyanobiphenyl-Modified Polyoxazolines}

The preparation of cyanobiphenyl compounds is represented in Scheme $1(1,2)$. To make new cyanobiphenyl compounds with different spacer length (1), 4-(4bromophenyl)phenol having biphenyl unit was first linked to halogen vinyl compounds having the different enes spacer $(n=4)$

\begin{tabular}{|c|c|c|c|c|}
\hline \multirow{2}{*}{ Run } & \multirow{2}{*}{$\begin{array}{l}\text { Copolymer } \\
q /(p+q)^{a}\end{array}$} & \multirow{2}{*}{$\frac{\text { Yield }}{\%}$} & \multicolumn{2}{|c|}{$y /(x+y)$} \\
\hline & & & ${ }^{1} \mathrm{H} \mathrm{NMR}^{\mathrm{b}}$ & $\mathrm{UV}^{\mathrm{c}}$ \\
\hline 1 & 0.03 & 51 & 0.06 & 0.05 \\
\hline 2 & 0.13 & 67 & 0.20 & 0.18 \\
\hline 3 & 0.27 & 54 & 0.34 & 0.35 \\
\hline 4 & 0.48 & 65 & 0.47 & 0.44 \\
\hline
\end{tabular}

p, q, x, y: see scheme 2. ${ }^{a}$ Determined by ${ }^{1} \mathrm{H}$ NMR. ${ }^{b}$ Determined by ${ }^{1} \mathrm{H}$ NMR (acetyl proton versus biphenyl proton). ${ }^{c} \mathrm{De}-$ termined by UV at $\lambda_{\max }=296 \mathrm{~nm}\left(\varepsilon=2.6 \times 10^{4}\right)$.

number of methylene units as the role of flexible spacer. The compounds having biphenyl unit were changed to the bromobiphenyl alcohol, and cyanobiphenyl alcohol through the hydroboration and cupper cyanide reaction in sequence. The resulting cyanobiphenyl alcohols were reacted with pyridinium dichromate (PDC) in DMF to give three kinds of cyanobiphenyl mesogenic compounds with different spacer which is di-, tetra-, and decamethylene. To make other cyanobiphenyl compound with hexamethylene spacer (2), the ethyl 7bromoheptanoate is used to link to the cyanobiphenyl group. 7-(4-Cyanobiphenyloxy)heptanoic acid (7) with hexamethylene spacer was prepared by the reaction of a base-promoted hydrolysis of esters and cupper cyanide in sequence. The obtained four kinds of cyanobiphenyl compounds (CB- $n, n=2,4,6,10$ ) were introduced to the four different partially hydrolyzed polyoxazolines in the presence of dicyclohexylcarbodiimide (DCC) as a condensing agent to give LC polyoxazolines having different contents of mesogens and spacer lengths. The solubility of the obtained LC polyoxazolines was in general poor in most organic solvents at room temperature. They have only a good solubility in chloroform and dichloromethane. There was no correlation between the length of spacer and solubility of all prepared LC polyoxazolines. Their solubility, however, decreased as the substitution degrees of cyanobiphenyl compounds increased.

Figure 1 shows ${ }^{1} \mathrm{H}$ NMR spectra of Poly 4-47 (degree of substitution, $47 \mathrm{~mol} \%$ by ${ }^{1} \mathrm{H}$ NMR) (9) and of the starting partially hydrozed polyoxazoline (degree of hydrolysis, $48 \mathrm{~mol} \%$ by ${ }^{1} \mathrm{H}$ NMR) (8). The peak of the methylene protons adjacent to the secondary amino group disappeared completely. At the same time, the peaks of cyanobiphenyl group and spacer methylenes of CB-4 ap- 

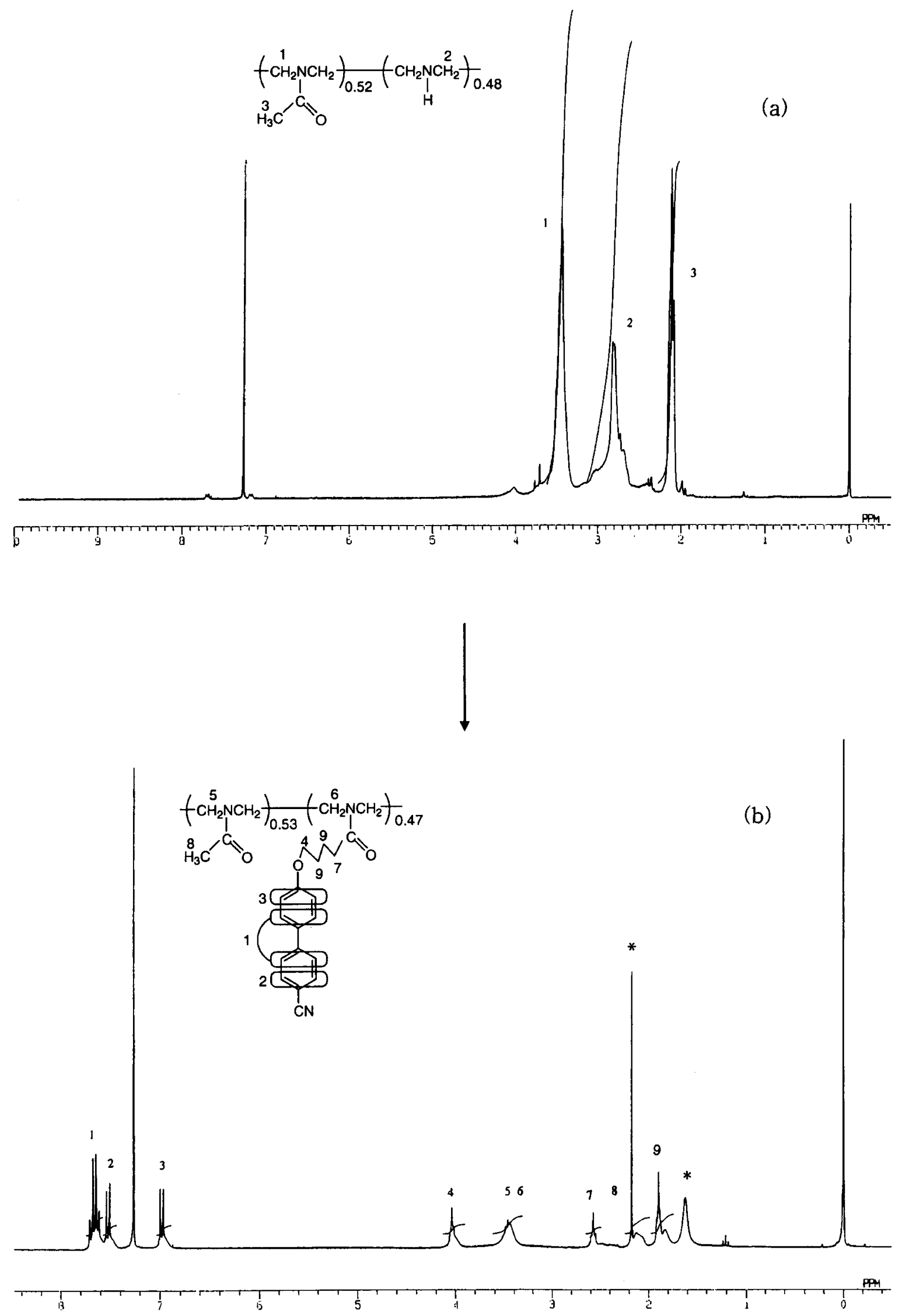

Figure 1. ${ }^{1} \mathrm{H}$ NMR spectra of (a) partially hydrolyzed polyoxazoline (48 mol\%) and (b) Poly 4-47. Asterisks show the peaks due to water and acetone, respectively. 

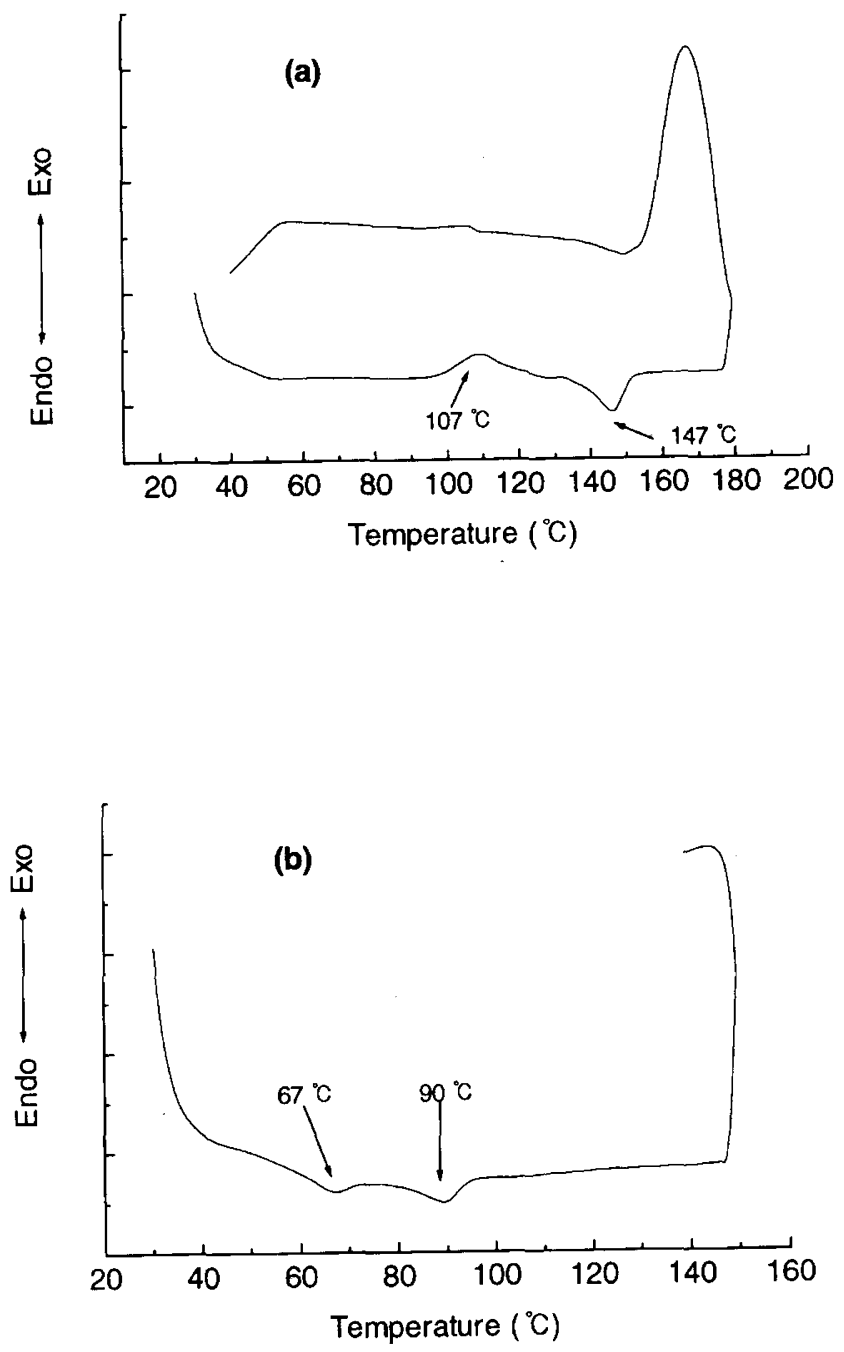

Figure 2. DSC thermograms of Poly 4-47 (a) and Poly 10-43 (b) obtained under a nitrogen atmosphere at the heating rate of $10^{\circ} \mathrm{C} \min ^{-1}$.

peared. The substitution degrees of CB-4 could be calculated by the integral ratio between the protons of biphenyl and the remaining $N$-acetyl protons. The degree of substitution could be also estimated from the UV spectrum of the LC polyoxazoline by using absorption coefficient of CB-4. Table I summarizes the results of the preparation of LC polyoxazolines with tetramethylene spacer together with their degrees of substitution. The substitution degrees estimated were well matched with the hydrolysis degrees in the starting partially hydrolyzed polyoxazolines. The substitution degrees of the other LC polyoxazolines were, to a certain extent, matched with the hydrolysis degrees in the starting partially hydrolyzed polyoxazoline.

\section{Thermal Property and Liquid Crystalline Behavior}

The results of the DSC investigation on the $\mathrm{LC}$ polyoxazolines with different spacer length (Poly 4-47, Poly 10-43) are shown in Figure 2. They only showed LC phase in the heating cycle. This stands for a typical monotropic LC behavior. In case of Poly 4-47, DSC curve was complicated unlike that of Poly 10-43; that is to say, a broad exotherm and a endotherm peak were detected at the same time on the heating cycle. It is considered
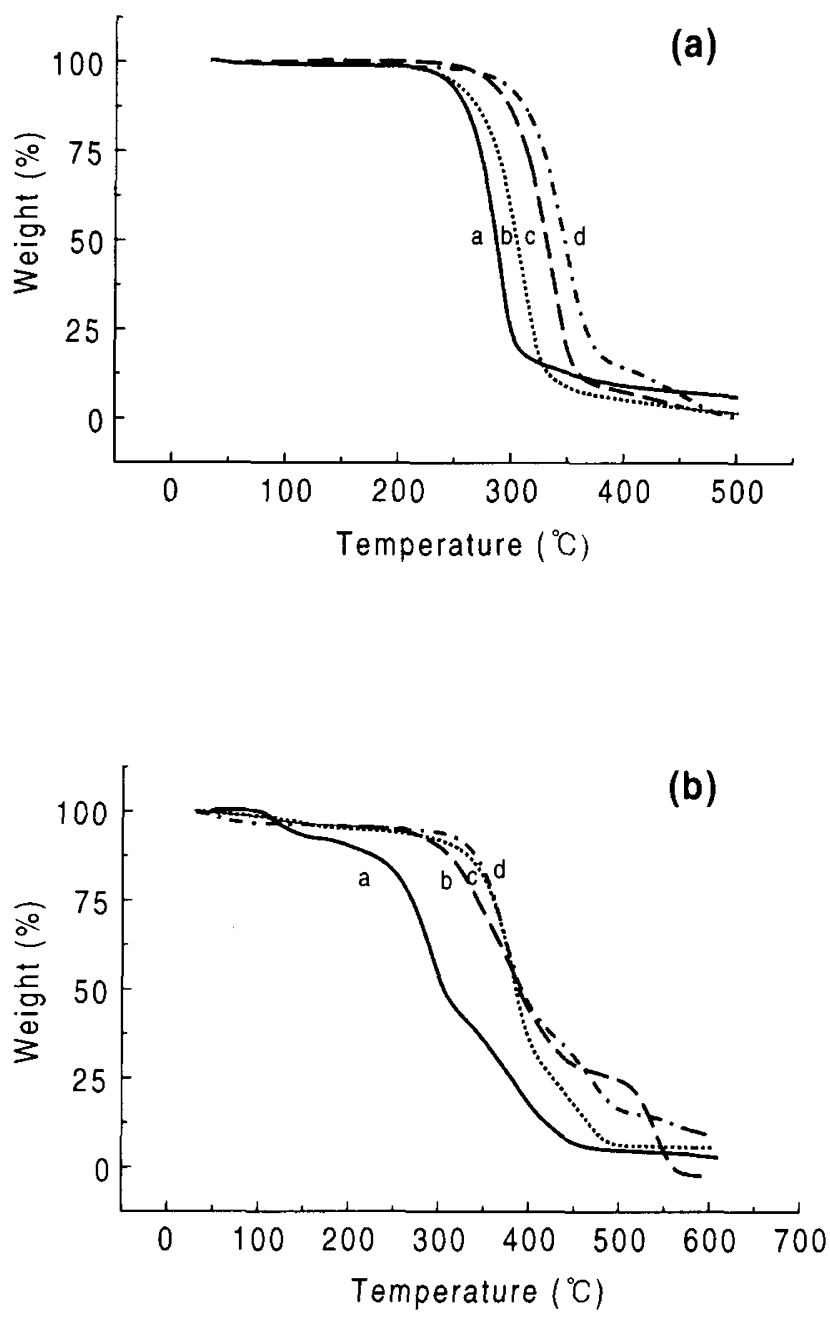

Figure 3. TGA thermograms of cyanobiphenyl compounds (a) and $\mathrm{LC}$ polyoxazolines $(\mathrm{b})$ with a heating rate of $10^{\circ} \mathrm{C} \mathrm{min}^{-1}$, (a): a, CB-2; b, CB-4; c, CB-6; d, CB-10, (b): a, Poly 2-55; b, Poly 4-47; c, Poly 6-53; $d$, Poly 10-43.

that after $T_{\mathrm{g}}$ which was not observed precisely in here, the orientation of a few mesogens resultig from the flexible and segmental motion of the polymer at the broad exotherm was immediately followed by the appearance of LC phase. The thermal stabilities of the cyanobiphenyl compounds and LC polyoxazolines were observed by TGA thermogram and shown in Figure 3. The temperature of $10 \%$ decomposition of cyanobiphenyl compounds was increased with increasing the length of methylene spacer $(2 \rightarrow 4 \rightarrow 8 \rightarrow 10)$. It means that thermal stability was increased. In case of the polyoxazolines having $47 \mathrm{~mol} \%$ of mesogens with different spacer length, the trend of decomposition temperature is same to that of cyanobiphenyl compounds. This may be ascribed to the increase in the flexibility as the length of spacer group is increased. ${ }^{4}$ In addition, the obtained polymers showed a good thermal property (10\% weight loss around $334^{\circ} \mathrm{C}$ : Poly $10-43$ ).

The LC behavior of the prepared cyanobiphenyl compounds and polyoxazolines having cyanobiphenyl moiety was investigated by means of DSC and optical polarizing microscopy. The phase transition temperatures of cyanobiphenyl compounds are summarized in Table II. All prepared cyanobiphenyl compounds show the monotropic 
Table II. Phase transition temperatures of cyanobiphenyl mesogenic compounds

\begin{tabular}{ccc}
\hline Spacer & \multicolumn{2}{c}{ Phase transition temperatures $/ /^{\circ} \mathrm{C}$} \\
\cline { 3 - 3 }$\left(\mathrm{CH}_{2}\right)_{n}$ & On cooling cycle ${ }^{\mathrm{a}}$ & $T_{\mathrm{m}}^{\mathrm{b}}$ \\
\hline$n=2$ & $\mathrm{i} 134 \mathrm{n} 114 \mathrm{k}$ & 167 \\
$n=4$ & $\mathrm{i} 118 \mathrm{n} 96 \mathrm{k}$ & 137 \\
$n=6$ & $\mathrm{i} 118 \mathrm{n} 89 \mathrm{k}$ & 119 \\
$n=10$ & $\mathrm{i} 109 \mathrm{n} 101 \mathrm{k}$ & 116 \\
\hline
\end{tabular}

a Observed by optical polarizing microscopy. ${ }^{b}$ Melting temperature was detected by DSC thermogram.

Table III. Phase transition temperatures of new LC polyoxazolines

\begin{tabular}{|c|c|c|}
\hline \multirow{2}{*}{ Polymer } & \multicolumn{2}{|c|}{ Phase transition temperatures $/{ }^{\circ} \mathrm{C}$} \\
\hline & On heating cycle ${ }^{a}$ & $T_{\mathrm{m}}^{\mathrm{b}}$ \\
\hline Poly 2-55 & g 140 s 163 & 163 \\
\hline Poly 2-35 & g 136 s 162 & 162 \\
\hline Poly 4-47 & g 107 s 147 & 147 \\
\hline Poly 4-34 & g $91 \mathrm{~s} 145$ & 145 \\
\hline Poly 6-53 & g 93 s 131 & 131 \\
\hline Poly 6-31 & g $85 \mathrm{~s} 130$ & 130 \\
\hline Poly $10-43$ & 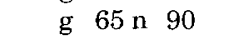 & 90 \\
\hline Poly 10-27 & g $82 \times 100$ & 100 \\
\hline
\end{tabular}

a Observed by optical polarizing microscopy. b Melting temperature was detected by DSC thermogram. ${ }^{*} M_{n}=6000-7000$ (determined by GPC, PSt standard).

liquid crystallinity in the cooling cycle at optical polarizing microscopy. This result is nearly consistent with that observed by DSC. The melting temperature and LC phase range of them were lowered as the length of the spacer group was increased. The obtained LC structure of all cyanobiphenyl compounds was found to be a typical nematic texture like thread-like and schlieren textures as shown in Figure 4.

The phase transition temperature of LC polyoxazolines are summarized in Table III. The phase transition temperatures and LC textures of LC polyoxazolines having low degrees of cyanobiphenyl mesogenic unit ( $3 \mathrm{~mol} \%$ or $13 \mathrm{~mol} \%$ ) could not be observed in both DSC and optical polarizing microscopy. It is confirmed that the critical percentage in which the LC phase can be shown is above $27 \mathrm{~mol} \%$ of cyanobiphenyl moiety introduced to hydrolyzed polyoxazolines. The melting temperature and LC phase range of them were lowered as the length of the spacer group was increased like the case of cyanobiphenyl compounds. These results may be attributed to the flexibility of the spacer which decouples the motion of side groups from that of the main chain of polyoxazoline. In other words, even the range of low temperature makes it possible to reorient or move cyanobiphenyl moiety as the spacer length is increased. Figure 2 shows DSC thermograms of Poly 4-47 and Poly 1043, respectively. As compared (a) with (b), LC phase range in (b) showed the lower range of temperature than that in (a). It is also observed that LC polyoxazolines have broader LC phase than the corresponding cyanobiphenyl compounds due to increase in the thermal stability of mesophase by polymer backbone. In case of LC polyoxazolines with di-, tetra-, and hexamethylene (a)

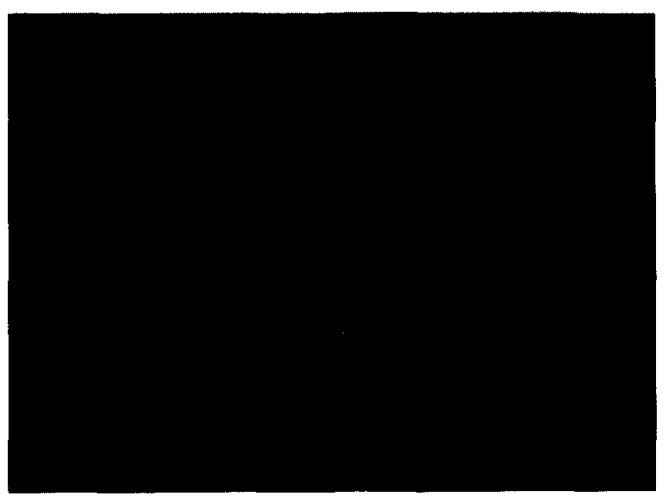

(b)

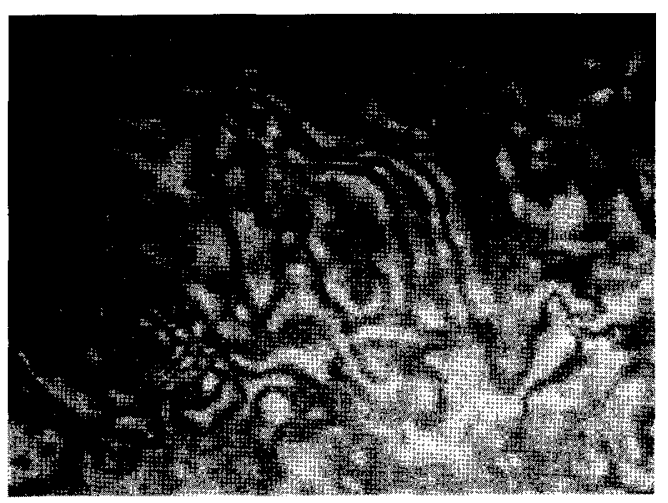

(c)

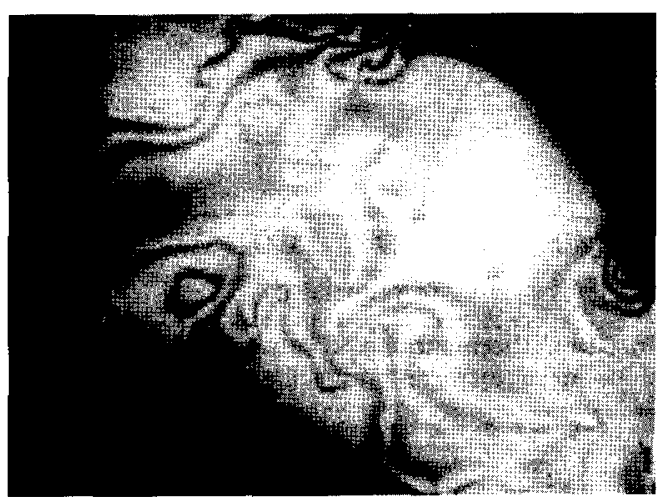

Figure 4. Optical polarizing micrographs of cyanobiphenyl compounds during a cooling cycle: $(a)(\times 100), \mathrm{CB}-4$ at $113^{\circ} \mathrm{C}$; (b) $(\times$ $100), \mathrm{CB}-6$ at $110^{\circ} \mathrm{C}$; (c) $(\times 200), \mathrm{CB}-10$ at $110^{\circ} \mathrm{C}$

spacer, the nematic texture of cyanobiphenyl compounds changed into smectic texture like bâtonnet and focalconic textures of the SCLCPs as attaching LC material to the polyoxazoline backbone. This proves that the polymer fixation of mesogenic groups stabilizes LC-phase. But, the LC structure of LC polyoxazolines with decamethylene spacer showed nematic texture without changing the texture of CB-10 as shown in Figure 5. It is considered that the effect of polymer backbone gradually decrease in the movement of cyanobiphenyl moiety due to the length of methylenes in the spacer.

\section{CONCLUSION}

Side-chain cyanobiphenyl polyoxazolines having different contents of mesogens and spacer lengths were synthesized and examined by DSC, TGA, and optical polarizing microscopy. The liquid crystallinity of all prepared cyanobiphenyl compounds shows nematic texture. These nematic textures were changed to the smectic tex- 
(a)

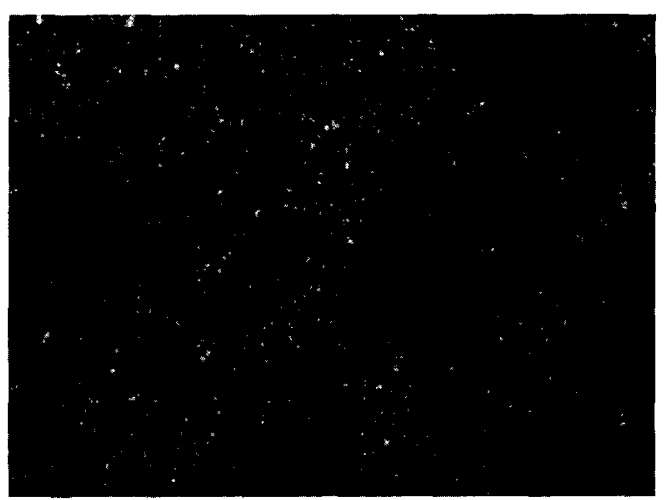

(c)

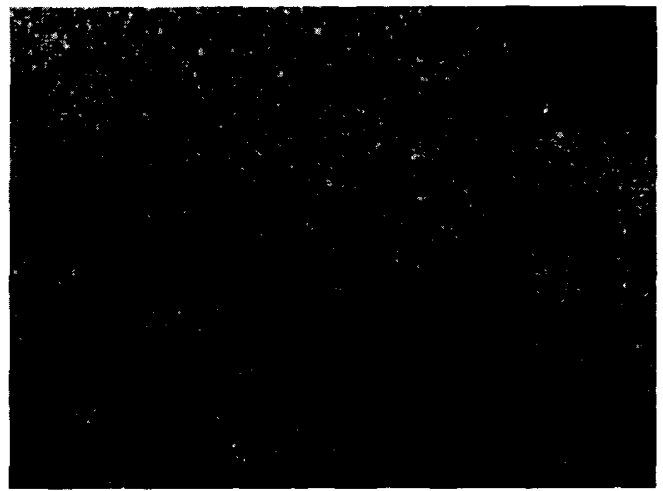

(e)

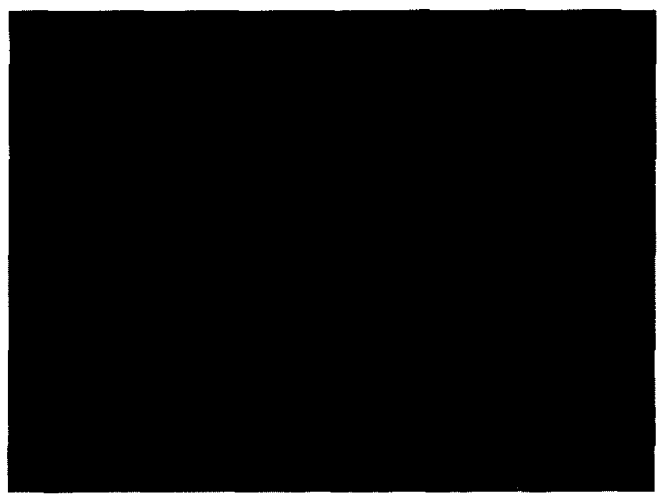

(b)

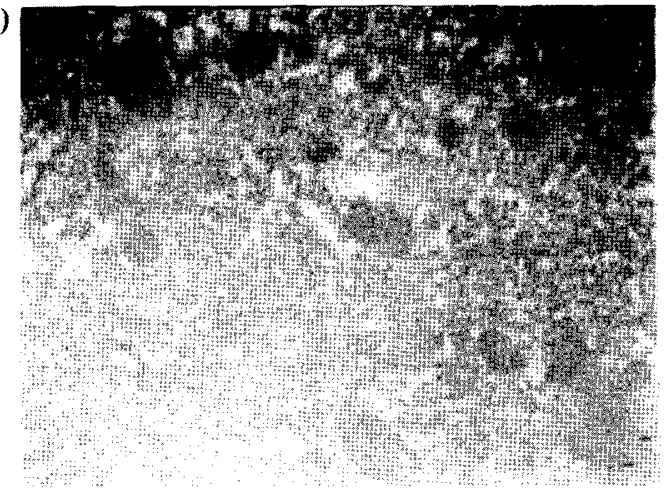

(d)

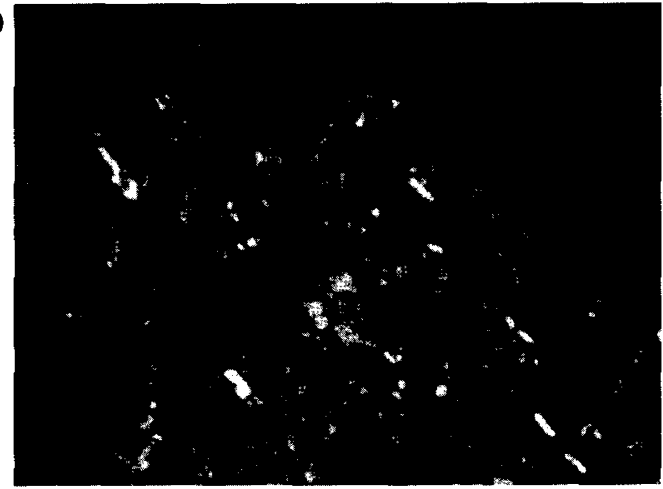

(f)

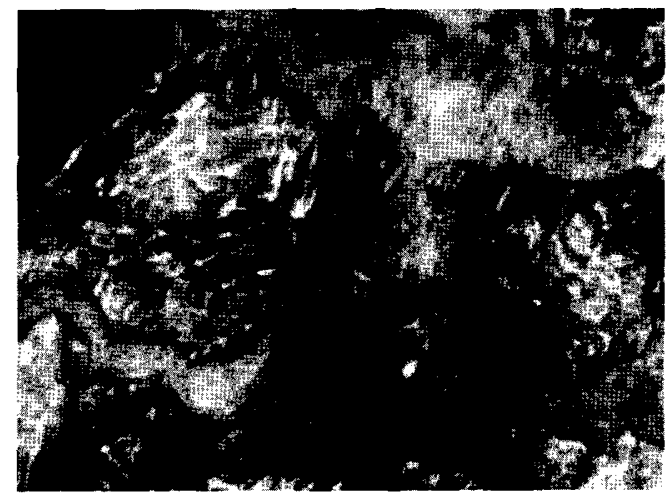

Figure 5. Optical polarizing micrographs of LC polyoxazolines during a heating cycle: (a), Poly $2-55$ at $142^{\circ} \mathrm{C}$; (b), Poly $4-34$ at $109^{\circ} \mathrm{C}$; (c), Poly $4-47$ at $134^{\circ} \mathrm{C}$; (d), Poly $6-31$ at $115^{\circ} \mathrm{C}$; (e), Poly $10-43$ at $81^{\circ} \mathrm{C}$; (f), Poly $10-43$ at $86^{\circ} \mathrm{C}$ (magnification $100 \times$ ).

ture of LC polyoxazolines except for LC polyoxazoline having decamethylene spacer. It was also found that LC phase of LC polyoxazolines was only shown above $27 \mathrm{~mol} \%$ of mesogens introduced to hydrolyzed polyoxazolines. Thus, we could conclude that their liquid crystallinities showed to be closely dependent on the degrees of substitution and the length of the spacer group incorporated into the portion of the ethylenimine. More experiments with LC homopolyoxazolines are in progress.

\section{REFERENCES AND NOTES}

1. H. Stegemeyer and Guest, Ed., "Liquid Crystals," Springer, New York, N.Y., 1994.

2. V. P. Shivaev, S. G. Kostromin, N. A. Plate, S. A. Ivanov, V. Y Vetrov, and I. A. Yakolev, Polym. Commun., 24, 364 (1983).

3. Y. K. Han, Y. D. Kim, and H. Y. Kim, J. Polym. Sci., Polym. Chem., 30, 1177 (1992).

4. R. H. Allcock and C. Kim, Macromolecules, 22, 2596 (1989).

5. X. L. Pia, J. S. Kim, Y. K. Yun, and J. I. Jin, Macromolecules, 30, 2294 (1997).

6. I. H. Cho and S. Y. Jo, Macromolecules, 32, 521 (1999).
7. P. Rochon, D. Bissonnette, A. Natansohn, and S. Xie, Applied Optics., 32, 35 (1993).

8. P. Rochon and A. Natansohn, Macromolecules, 29, 44 (1996).

9. S. Xie, A. Natansohn, and P. Rochon, Chem. Mater., 5, 403 (1993).

10. A. Natansohn, P. Rochon, J. Gosselin, and S. Xie, Macromolecules, 25, 5531 (1989).

11. S. Kobayashi, T. Igarashi, Y. Moriuchi, and T. Saegusa, Macromolecules, 19, 535 (1986).

12. M. Miyamoto, K. Aoi, and T. Saegusa, Macromolecules, 22 , 3540 (1989).

13. M. H. Litt, T. T. Chen, and B. R. Hsieh, J. Polym. Sci., Polym. Chem., 24, 3407 (1986).

14. Y. Chujo, Y. Yoshifuji, K. Sada, and T. Saegusa, Macromolecules, 22, 1074 (1989).

15. Y. Chujo, K. Sada, K. Matsumoto, and T. Saegusa, Polym. Bull., 21, 353 (1989).

16. Y. Chujo, K. Sada, K. Matsumoto, and T. Saegusa, Macromolecules, 23, 1234 (1990).

17. M. Miyamoto, K. Naka, M. Shiozaki, Y. Chujo, and T. Saegusa, Macromolecules, 23, 3201 (1990).

18. K. Naka, Y. Kubo, A. Ohki, and S. Maeda, Polym. J., 26, 243 (1994).

19. Y. Chujo, K. Sada, and T. Saegusa, Macromolecules, 23, 2693 
(1990).

20. Y. Imai, K. Naka, and Y. Chujo, Polym. J., 30, 990 (1998).

21. T. Saegusa and Y. Chujo, Makromol. Chem., Macromol. Symp., 33, 31 (1990)

22. S. Kobayashi, M. Kaku, and T. Saegusa, Macromolecules, 21, 334 (1988).
23. J. M. Rodriguez-Parada and V. Percec, J. Polym. Sci., Polym. Chem. Ed ., 25, 2269 (1987)

24. G. P. Ellis and T. M. Romney-Alexander, Chem. Rev., 87, 779 (1987).

25. E. J. Corey and G. Schmidt, Tetrahedron Lett., 5, 399 (1979), 